\title{
Efficacy of adjuvant radiotherapy for treatment of adrenocortical carcinoma: a retrospective study and an updated meta-analysis
}

Jiawei Zhu

Chinese Academy of Medical Sciences and Peking Union Medical College

\section{Ziye Zheng}

Chinese Academy of Medical Sciences and Peking Union Medical College https://orcid.org/0000-00020545-2781

\section{Jing Shen}

Peking Union Medical College Hospital

\section{Xin Lian}

Peking Union Medical College Hospital

\section{Zheng Miao}

Peking Union Medical College Hospital

Jie Shen ( $13521039164 @ 163 . c o m$ )

https://orcid.org/0000-0002-3845-2040

\section{Fuquan Zhang}

Peking Union Medical College Hospital

\section{Research}

Keywords: adrenocortical carcinoma, adjuvant radiotherapy, surgery, meta-analysis

Posted Date: March 11th, 2020

DOI: https://doi.org/10.21203/rs.3.rs-16735/v1

License: (c) (1) This work is licensed under a Creative Commons Attribution 4.0 International License.

Read Full License

Version of Record: A version of this preprint was published at Radiation Oncology on May 24th, 2020. See the published version at https://doi.org/10.1186/s13014-020-01533-3. 


\section{Abstract}

Background Adrenocortical carcinoma (ACC) is a rare and highly aggressive malignancy. Surgical resection is the standard treatment for localized ACC, but the local recurrence remains high. Adjuvant radiation (ART) has been proposed as a means to reduce recurrence rates in ACC after surgery with conflicting results from nonrandomized studies. We performed a retrospective study and a meta-analysis to determine the impact of ART on survival outcomes. Methods A retrospective study of the adrenocortical cancer database in Peking Union Medical College was conducted. We selected postoperative ACC patients with or without ART. A meta-analysis is also performed to compare the outcomes between ART and only surgical resection in ACC patients. The obtained data were analyzed using SPSS 23.0 and Stata 15.0 statistical software. Differences between two groups were compared using the log-rank test for retrospective analysis and estimated by calculating the odds ratio (OR) and 95\% confidence interval (Cl) for meta-analysis. Results Of a total of 75 patients available in the database, 12 patients underwent postoperative ART and were matched one to one to patients with only surgical resection. There was no significant difference on overall survival between ART group and control group (log-rank $P=0.149$ ). Locoregional recurrence was diagnosed in 2 of the ART group, and in 4 of the control group $(P=0.64)$. A total of 238 participants were selected for the meta-analysis, of which 111 and 127 patients underwent ART after surgical resection and only surgical resection, respectively. Overall survival is significantly higher in ART group, with an odds ratio (OR) of $2.41(95 \% \mathrm{Cl}$ of $1.33,4.38 ; \mathrm{P}=$ 0.004). Besides, meta-analysis significantly favored ART for locoregional recurrence-free survival and disease-free survival, with an OR of 4.08 and 2.27, respectively. Conclusions Our results show that compared to only surgical resection, ART is an effective postoperative treatment for ACC.

\section{Background}

Adrenocortical carcinoma (ACC) is a rare and highly aggressive malignancy with an incidence rate of 1-2 case per million population [1]. Surgical resection is the standard treatment for localized ACC to achieve a long-term survival [2]. However, locoregional failures are common even after complete surgical resection with estimated rates of local recurrence as high as 30\% [3], which was with associated poor long-term survivorship [4]. Distant failures are also quite often, with $42 \%$ patients having liver metastases and $39 \%$ patients having liver metastases at initial stage or during follow-up [5].

Adjuvant treatments including mitotane and radiotherapy (RT) can extend tumor progression time. Adjuvant radiation (ART) has been proposed as a means to reduce high recurrence rates in ACC following surgery with curative intent [6]. A recent population-based study used the National Cancer Database and found that ART on improved overall survival (OS) with a $40 \%$ decreased risk of death in patients with positive margins [6]. Due to the rarity of this disease, there is a paucity of high-quality prospective evidence in academic literature evaluating the role of ART in ACC. Therefore, physicians must rely on the critical appraisal of non-randomized studies with variable patient populations, clinical stage, and outcomes. 
In order to improve clinical decision making, we systematically reviewed the literature for studies evaluating the role of ART after primary surgery of ACC, and performed a meta-analysis of oncological outcomes including OS, locoregional recurrence-free survival (LRFS), and recurrence-free survival (RFS). Although previous reviews have investigated this question, our systematic review provides an updated analysis. We excluded single arm studies from our review to provides a current and rigorous review of this question. We also report our clinical experience with ACC by comparing patients who were treated using surgery followed by ART with those using surgery alone.

\section{Methods}

\section{Institutional paired match comparison}

We performed a retrospective search of our adrenocortical cancer database. We selected data from adult patients who were treated using surgery, between 1 January 1994 and 1 January 2014.

The patients were separated into two Groups for analysis based on treatment strategy. The treatment group consisted of individuals treated with surgery followed by ART; the control group consisted of individuals treated with surgery only. The inclusion criteria comprised reported surgical margins, ART performed at our institution. The periods of use and doses given were inconsistent among patients because of variation in tolerance and toxicity. The exclusion criteria comprised patients that underwent ART for the treatment of recurrence or of secondary lesions, intra-operative rupture of the tumor capsule. The required minimal follow-up periods were 1 years after surgery, except for the patients that died before. The two groups were paired for comparison based on age, sex, surgical margin status, tumor site, ki67 index, mitotane, tumor size, and clinical stage according to European Network for the Study of Adrenal Tumors classification guidelines. Conformal three-dimensional radiotherapy was applied at the tumor bed for all patients in the ART group within 2 months after surgery, except one patient had ART within 6 months after surgery. Radiation extension and dose were chosen according to adrenocortical cancer severity (based on margin status, size, stage, and immunohistochemistry).

The groups were compared using OS, LRFS, and RFS outcome variables. Local and distant recurrences were diagnosed using computed tomography magnetic resonance or positron-emitting tomography imaging. The times to local and distant recurrence were considered the periods from the surgery date to the date of the imaging examination that revealed the recurrence. If recurrence did not occur, patients were censored at the date of death or at the date of the last follow-up examination. Two and one patients with oligometastatic disease were included in ART group and control group, respectively. These three patients were excluded from the disease-free survival analysis. OS time was measured from the surgery date to the date of death. Patients still living at the last date of follow-up were censored in the analysis.

The statistical analysis was performed using SPSS 23.0. The two groups were compared using the paired $\mathrm{t}$ test for continuous variables and Fisher's exact test for categorical variables. The Kaplan-Meier method was used for the survival analysis (GraphPad Prism 8.02 for Windows application). The differences 
between two groups in endpoints were compared using the log-rank test. All tests were 2-sided. A p-value $<0.05$ was considered to indicate a statistically significant result.

\section{Meta-analysis for oncological outcomes}

This study was conducted and reported according to the Preferred Reporting Items for Systematic Reviews and Meta-Analysis Statement (PRISMA) issued in 2009 [7]. The PRIMA checklist is attached (S1 File). A systematic electronic search of PubMed, EmBase, and the Cochrane Library databases was performed for eligible studies from inception to September 2019. Studies that compared the outcomes between ART and only surgical resection for the treatment of ACC were selected for meta-analysis in this study. There is no language or publication status limitation for literature review. The following index words were used: adrenocortical, carcinoma, cancer, radiation, radiotherapy, irradiation and adjuvant.

The retrieved records were independently reviewed by two investigators. If the investigators disagreed about the eligibility of an article, it was resolved by debating with a third reviewer. The inclusion criteria were as follows: (1) patients with adrenocortical carcinoma; (2) intervention was ART compared with only surgical resection; (3) outcomes data reported; and (4) article type as original research. In the current study, the outcome included tumor outcomes, such as OS, LRFS and RFS.

The Newcastle-Ottawa Scale (NOS) was used to evaluate the quality of the included studies for cohort studies. The NOS was based on the following three subscales: selection (four items), comparability (one item), and outcome (three items). NOS ranges were 0 to 8 , and scores of $0-3,4-6$, and 7-8 were considered as low, moderate, and high quality, respectively, for these studies.

Event numbers of oncologic outcomes from both ART and control group were pooled from eligible studies. Meta-analysis was performed using Stata software (version 15.0; Stata Corporation, College Station, TX, USA). Heterogeneity between studies was assessed via a chi-square analysis and the I [8] test, with significance set at $\mathrm{P}<0.05$. Included studies are considered to have low heterogeneity (or be homogeneous) if I [8] is less than 25\%, moderate heterogeneity if I [8] is 25 to $50 \%$, and high heterogeneity if I [8] is greater than $50 \%$. If homogeneity existed between studies, meta-analysis was performed with a fixed effect model. If significant heterogeneity was confirmed, either by significant chi-square test $(P<$ 0.05) or I [8] greater than $50 \%$, meta-analysis was performed using a random effects model. Lastly, a pooled odds ratio (OR) was performed with 95\% confidence interval $(\mathrm{Cl})$, and the overall effect was assessed via the $z$ statistic with statistical significance set at $P<0.05$.

\section{Results}

\section{Institutional paired match comparison}

A total of 75 patients were available to assess from the institutional database. Of these, 12 patients underwent postoperative adjuvant RT at the Peking Union Medical College Hospital. These were matched one to one to patients who did not undergo adjuvant RT suing the propensity matching. Patients 
underwent resection between 2003 and 2018. All patients had localized or oligometastatic disease and underwent surgery with curative intent. Median follow-up times for the ART and control groups were 23 months (range: 10-76) and 37 months (14-92). respectively.

Table 1 summarizes the baseline characteristics of patients by treatment group. There was no significant difference between the groups in term of sex, age, stage, receipt of mitotane, tumor size, endocrine syndrome, or surgical margin status. The majority of patients treated with adjuvant RT were treated after 2010 (91.7\%). There was an average of 51 days from the date of surgery to initiation of RT (range, 16 to 199 days). Only one patient had a more than 100 days interval between surgery and initiation of RT. All patients were treated with three-dimensional conformal RT.

The OS distributions for case subjects and control subjects were significantly different (log-rank $\mathrm{P}=$ 0.149) (Fig. 1A). A total of $9(58.3 \%)$ patients [ $5(41.7 \%)$ treated with adjuvant RT and 4 (33.3\%) treated without adjuvant RT] are known to have died. OS estimates at 3 were $62.7 \%$ vs $71.0 \%$, respectively, with an adjusted $\mathrm{HR}$ of $2.81(95 \% \mathrm{Cl}, 0.65$ to $12.09 ; \mathrm{P}=0.165)$.

Local recurrence was diagnosed in 2 of the ART group, and in 4 of the control group ( $P=0.64)$. Locoregional recurrence-free survival (LRFS) was similar between the two groups (log-rank $P=0.879$ ) (Fig. 1B). Locoregional RFS estimates at 3 years were $73.6 \%$ vs $56.6 \%$, respectively, with an adjusted HR of $0.87(95 \% \mathrm{Cl}, 0.16$ to $4.91 ; \mathrm{P}=0.88)$.

Any recurrence, including distant failures or death, occurred in $15(71.4 \%)$ patients [7 (70\%) treated with RT and 8 (72.7\%) treated with resection alone]. There was an insignificant difference in DFS between patients who received adjuvant RT and those who underwent resection only (log-rank P value 0.085) (Fig. 1C). DFS estimates at 3 years were $37.5 \%$ vs $60.3 \%$, respectively, with an adjusted $\mathrm{HR}$ of $2.58(95 \% \mathrm{Cl}$, 0.846 to $7.84 ; P=0.096)$.

\section{Meta-analysis for oncological outcomes}

A total of 191 studies were retrieved based on our searching strategy. After reading the abstract, 16 studies were related to our aims (Fig. 2), and 11 of them were subsequently excluded with reasons give. The remaining 5 studies and our data were selected for our analysis (Table 2) [9-13]. A total of 238 participants were selected for the meta-analysis, of which 111 and 127 patients underwent ART after surgical resection and only surgical resection, respectively. All the studies included were retrospective design and the majority of them were from Europe and America.

The Newcastle-Ottawa Scale (NOS) was used to examine the quality of all included studies. Most studies (5 of 7) were marked as 7 in NOS as they failed to report enough follow-up time. The 1 remaining study received full scores in NOS, indicating they were high quality original studies (Table 3 ).

\section{Overall survival}


Of the six studies included in our meta-analysis, five reported OS event numbers for both ART group and control group (Fig. 3). The total patient population was 95 in both groups. There was significant heterogeneity among the five retrospective studies $\left(\mathrm{Chi}^{2}=7.81, P=0.099, \mathrm{I}^{2}=48.8 \%\right)$, and the fixed effects model was applied. Meta-analysis significantly favored OS with ART, with an OR of $2.41(95 \% \mathrm{Cl}$ of $1.33,4.38 ; P=0.004)$.

\section{Local recurrence-free survival}

All the six studies reported event numbers for LRFS for both ART and control groups (Fig. 4). There were 111 patients in the ART group and 127 in the control group. Meta-analysis revealed significant heterogeneity among the six retrospective studies $\left(\mathrm{Chi}^{2}=15.83, P=0.007, \mathrm{I}^{2}=68.4 \%\right)$, and the random effects model was applied. The pooled analysis significantly favored ART for LRFS of ACC patients, with an OR of 4.08 (95\% $\mathrm{Cl}$ of $1.29,2.14 ; P=0.016)$. After performing a sensitive analysis, and found the heterogeneity was introduced by the MDACC study [10]. The imbalanced sample size and different follow-up time caused the referral bias. After that, combining the results of the five cohorts (190 patients) a statistical significance in favor of ART group was observed with a OR $=6.25$ (95\% IC 3.24-12.05, $P<$ 0.001 ) (Fig. 5). There was low heterogeneity among the five studies $\left(\mathrm{Chi}^{2}=5.69, \mathrm{P}=0.224, \mathrm{I}^{2}=29.6 \%\right.$ ) indicating that the pooled analysis is valid.

\section{Recurrence-free survival}

Five of six studies reported event numbers for recurrence-free survival for both ART and control groups (Fig. 6). There were 92 patients in the ART group and 93 in the control group. There was low heterogeneity among the five retrospective studies $\left(\mathrm{Chi}^{2}=3.79, P=0.435, \mathrm{I}^{2}=0 \%\right)$, and the fixed effect model was applied. Meta-analysis significantly favored ART for disease-free survival in ACC, with an OR of 2.27 (95\% Cl of 1.23, 4.18; $P=0.009$ ).

\section{Discussion}

ACC was historically thought to be radioresistant due to the mixed results from previous case reports or case series publish between 1958 and 2006[14-16]. With the application of modern radiation techniques and the publication of high-quality retrospective studies, the view of the role of ART in the treatment of ACC has changed. Besides, the use of mitotane showed to have a beneficial effect as adjuvant treatment after radical resection of primary ACC [17]. Experimental study suggested that mitotane may a sensitizer for radiotherapy [18] and most included articles for this meta-analysis have comprised patients using it. Gharzai et al. performed a retrospective propensity-matched analysis on 39 patients of ACC treated with ART. Results showed that LRFS and OS was significantly better with ART, compared with surgery only.

The results for our study cohort showed there were no significant difference between ART group and control group. However, we are the first systematic review comparing ART after resection and resection 
only in ACC to demonstrate a significant improvement in OS with ART (Fig. 3). In addition, our study shows that ART is strongly favorable for LRFS and RFS, with ORs of 4.08 and 2.27 respectively (Fig. 4\&6). With ART after primary surgery, patients are therefore approximately four times more likely to be local recurrence-free than those treated with surgery only. This finding has the same results with two previous systematic reviews with meta-analyses $[12,16]$. In terms of recurrence-free survival, debate exists as to whether ART after surgery is advantageous as compared to surgery only. One systematic review supported this notion [12], while the other does not find a significant difference [16]. With the inclusion of recent studies and our data, we demonstrate that treatment with ART is associated with improved recurrence-free survival in ACC. Overall, our results confirm the advantage of ART after primary surgery in OS, LRFS and RFS.

The advantages result from the progress in RT technique and machine, such as accurate delivery of disease site, and precise and consistent coverage of high-risk areas. Considering $25-30 \%$ of ACC patient may have inter-aortocaval lymph node metastases [19], this advance is beneficial to irradiate a wide territory. Besides, the RT adverse effects were considered tolerable and self-limited in all included studies. The main acute toxicity was nausea, fatigue, diarrhea with a low rate of potential kidney damage [12].

Regarding the ART dose, all the six retrospective studies used a median dose $\geq 50$ Gy with a dose of 1.82.0 Gy per fraction (Fig. 7). The majority of patients was treated with conformal three-dimensional RT with only two patients reported to treated with conventional RT in the Germany study [9]. Two included studies did not describe the details on treatment technique, target volume, or treatment machine $[10,12]$. It was difficult to observe a relation between the LRFS rate with RT dose or RT technique based on the limited available evidence.

The present study had a few limitations. Firstly, it is limited by its retrospective design and small sample size. Secondly, there were no available RCTs that evaluate ART in the treatment of ACC, which introduced selection bias. Thirdly, RT techniques and treatment plans were different among included studies, which may lead to heterogeneity in the meta-analysis. Last but not least, the lack of detailed information on RT method also increased the heterogeneity observed. Given the rarity of ACC and the limited literature on the use of ART, our work represented high-quality evidence.

\section{Conclusions}

Patients who had under gone ART after primary resection have increased OS, LRFS and RFS compared to resection only. Our results suggest that ART may be a beneficial treatment modality for ACC because of the improved survival outcome. However, a larger-scale RCT would be urgently needed to prove these advantages.

\section{Abbreviations}


ACC: Adrenocortical carcinoma; OR: odds ratio; RT: radiotherapy; ART: Adjuvant radiation; OS: Overall survival; RFS: recurrence-free survival; LRFS: Locoregional recurrence-free survival;

\section{Declarations}

\section{Ethics approval and consent to participate}

The Institutional Review Board (IRB) of Peking Union Medical College Hospital (PUMCH) reviewed the protocol. This is retrospective and meta-analysis study. The protocol is rational and scientific. The study accords with principle of ethics. The IRB thus approve the protocol. This is a retrospective study and written human subject consent was unnecessary.

\section{Consent for publication}

No applicable, as no individual patient data has been published.

\section{Availability of data and materials}

Owing to data privacy policy at our facility, publication of patient-related raw data is not possible.

\section{Competing interests}

The authors have no conflict of interest to disclose.

\section{Funding}

No funding was received.

Author Contributions: JW.Z and ZY.Z collected, analyzed, and interpreted the data; conducted the metaanalysis; wrote the manuscript. Jing.S, X.L, Z.M and FQ.Z reviewed and edited the manuscript. Jie.S designed the study, interpreted the data, critically reviewed the manuscript, and supervised the study. Jie.S has full access to all the data in the study and final responsibility for the decision to submit for publication. All authors read and approved the final manuscript.

\section{Acknowledgements}

We appreciate the support of PUMCH and the effort of every medical stuff who have treated these patients.

\section{References}

1. Kerkhofs, T.M.A., et al., Adrenocortical carcinoma: a population-based study on incidence and survival in the Netherlands since 1993. European journal of cancer (Oxford, England : 1990), 2013. 49(11): p. 2579-2586. 
2. Fassnacht, M., et al., European Society of Endocrinology Clinical Practice Guidelines on the management of adrenocortical carcinoma in adults, in collaboration with the European Network for the Study of Adrenal Tumors. European journal of endocrinology, 2018. 179(4).

3. Else, T., et al., Adrenocortical carcinoma. Endocrine reviews, 2014. 35(2): p. 282-326.

4. Tran, T.B., et al., Actual 10-year survivors following resection of adrenocortical carcinoma. Journal of surgical oncology, 2016. 114(8): p. 971-976.

5. Abiven, G., et al., Clinical and biological features in the prognosis of adrenocortical cancer: poor outcome of cortisol-secreting tumors in a series of 202 consecutive patients. The Journal of clinical endocrinology and metabolism, 2006. 91(7): p. 2650-2655.

6. Nelson, A.A. and G. Woodard, Severe adrenal cortical atrophy (cytotoxic) and hepatic damage produced in dogs by feeding 2,2-bis(parachlorophenyl)-1,1-dichloroethane (DDD or TDE). Arch Pathol (Chic), 1949. 48(5): p. 387-94.

7. Moher, D., et al., Preferred reporting items for systematic reviews and meta-analyses: the PRISMA statement. PLoS Med, 2009. 6(7): p. e1000097.

8. Tan, A. and D.A. Glass, 2nd, Patient-reported outcomes for keloids: a systematic review. G Ital Dermatol Venereol, 2019. 154(2): p. 148-165.

9. Fassnacht, M., et al., Efficacy of adjuvant radiotherapy of the tumor bed on local recurrence of adrenocortical carcinoma. J Clin Endocrinol Metab, 2006. 91(11): p. 4501-4.

10. Habra, M.A., et al., A retrospective cohort analysis of the efficacy of adjuvant radiotherapy after primary surgical resection in patients with adrenocortical carcinoma. J Clin Endocrinol Metab, 2013. 98(1): p. $192-7$.

11. Sabolch, A., et al., Adjuvant radiation therapy improves local control after surgical resection in patients with localized adrenocortical carcinoma. Int J Radiat Oncol Biol Phys, 2015. 92(2): p. 252-9.

12. Srougi, V., et al., Adjuvant radiotherapy for the primary treatment of adrenocortical carcinoma: Are we offering the best? Int Braz J Urol, 2017. 43(5): p. 841-848.

13. Gharzai, L.A., et al., Adjuvant Radiation Improves Recurrence-Free Survival and Overall Survival in Adrenocortical Carcinoma. J Clin Endocrinol Metab, 2019. 104(9): p. 3743-3750.

14. Macfarlane, D.A., Cancer of the adrenal cortex; the natural history, prognosis and treatment in a study of fifty-five cases. Annals of the Royal College of Surgeons of England, 1958. 23(3): p. 155-186.

15. Hutter, A.M. and D.E. Kayhoe, Adrenal cortical carcinoma. Clinical features of 138 patients. The American journal of medicine, 1966. 41(4): p. 572-580.

16. Viani, G.A. and B.S. Viana, Adjuvant radiotherapy after surgical resection for adrenocortical carcinoma: A systematic review of observational studies and meta-analysis. Journal of cancer research and therapeutics, 2019. 15(Supplement): p. S20-S26.

17. Terzolo, M., et al., Adjuvant mitotane treatment for adrenocortical carcinoma. The New England journal of medicine, 2007. 356(23): p. 2372-2380. 
18. Cerquetti, L., et al., Mitotane increases the radiotherapy inhibitory effect and induces G2-arrest in combined treatment on both H295R and SW13 adrenocortical cell lines. Endocrine-related cancer, 2008. 15(2): p. 623-634.

19. Livhits, M., et al., Surgery is associated with improved survival for adrenocortical cancer, even in metastatic disease. Surgery, 2014. 156(6).

\section{Tables}

Table 1. Baseline Characteristics of Patients

\begin{tabular}{|c|c|c|c|}
\hline & ART (n=12) & Only Surgery $(n=12)$ & $P$ value \\
\hline Sex, n (\%) & & & \multirow{3}{*}{$1^{\mathrm{a}, \mathrm{c}}$} \\
\hline Male & $5(41.7)$ & $5(41.7)$ & \\
\hline Female & $7(58.3)$ & $7(58.3)$ & \\
\hline Mean age, y (range) & $43.4(19-70)$ & $42.9(22-62)$ & $0.924^{\mathrm{b}, \mathrm{c}}$ \\
\hline \multicolumn{4}{|l|}{ Disease stage } \\
\hline I & $2(16.7)$ & $1(8.3)$ & \multirow[t]{4}{*}{$1^{\mathrm{a}, \mathrm{c}}$} \\
\hline II & $5(41.7)$ & $6(50.0)$ & \\
\hline IIII & $3(25.0)$ & $4(33.3)$ & \\
\hline IV & $2(16.7)$ & $1(8.3)$ & \\
\hline Mitotane use & & & \\
\hline Yes & $4(33.3)$ & $3(25.0)$ & \multirow[t]{2}{*}{$1^{\mathrm{a}, \mathrm{c}}$} \\
\hline No & $8(66.7)$ & $9(75.0)$ & \\
\hline $\begin{array}{l}\text { Ki67 index } \\
<20 \%\end{array}$ & $4(33.3)$ & $5(41.7)$ & \multirow{2}{*}{$0.772^{\mathrm{a}, \mathrm{c}}$} \\
\hline $\begin{array}{l}\geq 20 \% \\
\text { Not reported }\end{array}$ & $\begin{array}{l}5(41.7) \\
3(25.0)\end{array}$ & $\begin{array}{l}3(25.0) \\
4(33.3)\end{array}$ & \\
\hline Tumor side & & & \\
\hline Right & $8(66.7)$ & 7 (58.3) & \multirow[t]{2}{*}{$1^{\mathrm{a}, \mathrm{c}}$} \\
\hline Left & $4(33.3)$ & $5(41.7)$ & \\
\hline Mean tumor size, cm (range) & $7.9(3-15.3)$ & $8.4(4.5-16.5)$ & $0.688^{\mathrm{b}, \mathrm{c}}$ \\
\hline $\begin{array}{l}\text { Surgical margins } \\
\text { Neqative }\end{array}$ & & & \multirow{3}{*}{$0.848^{\mathrm{a}, \mathrm{c}}$} \\
\hline $\begin{array}{l}\text { Negative } \\
\text { Positive }\end{array}$ & $\begin{array}{l}6(50.0) \\
4(33.3)\end{array}$ & $\begin{array}{l}8(66.7) \\
2(16.7)\end{array}$ & \\
\hline Not reported & $2(16.7)$ & $2(16.7)$ & \\
\hline
\end{tabular}

${ }^{a}$ Fisher's exact test.

${ }^{b}$ Paired $t$ test.

${ }^{\mathrm{c}}$ Accounted for in the model calculating the propensity weights for adjuvant RT.

Table 2. Characteristics of the included studies

\begin{tabular}{|c|c|c|c|c|c|c|c|c|}
\hline Study. year & Country & Study types & $\begin{array}{l}\text { Age (RT vs } \\
\text { non RT, } \\
\text { years) }\end{array}$ & $\begin{array}{l}\text { Gender (LS } \\
\text { vs RT, male } \\
\% \text { ) }\end{array}$ & $\begin{array}{l}\text { RT } \\
\text { participants }\end{array}$ & $\begin{array}{l}\text { Non RT } \\
\text { participants }\end{array}$ & Disease stage & $\begin{array}{l}\text { Follows-up } \\
\text { (LS vs RT, } \\
\text { month) }\end{array}$ \\
\hline Habra, 2012 & America & Retrospective & 48 vs 44 & 37.5 vs 34.4 & 16 & 32 & II and III & $\begin{array}{l}22.08 \mathrm{vs} \\
33.24(1.84 \mathrm{yr} \\
\text { vs } 2.77 \mathrm{yr})\end{array}$ \\
\hline Srougi, 2017 & Brazil & Retrospective & 40 v 38 & 40 vs 10 & 10 & 10 & $\mathrm{~N} / \mathrm{A}$ & 32 vs 35 \\
\hline Gharzai, 2019 & America & Retrospective & 44.9 vs 47.1 & 46.2 vs 46.2 & 39 & 39 & I, II, III and IV & $50.52(4.21 \mathrm{yr})$ \\
\hline Zhu, 2020 & China & Retrospective & 43.4 vs 42.9 & 41.7 vs 41.7 & 12 & 12 & I, II, III and IV & 23 vs 36.9 \\
\hline
\end{tabular}


Table 3. Methodological quality of the studies included in the meta-analysis

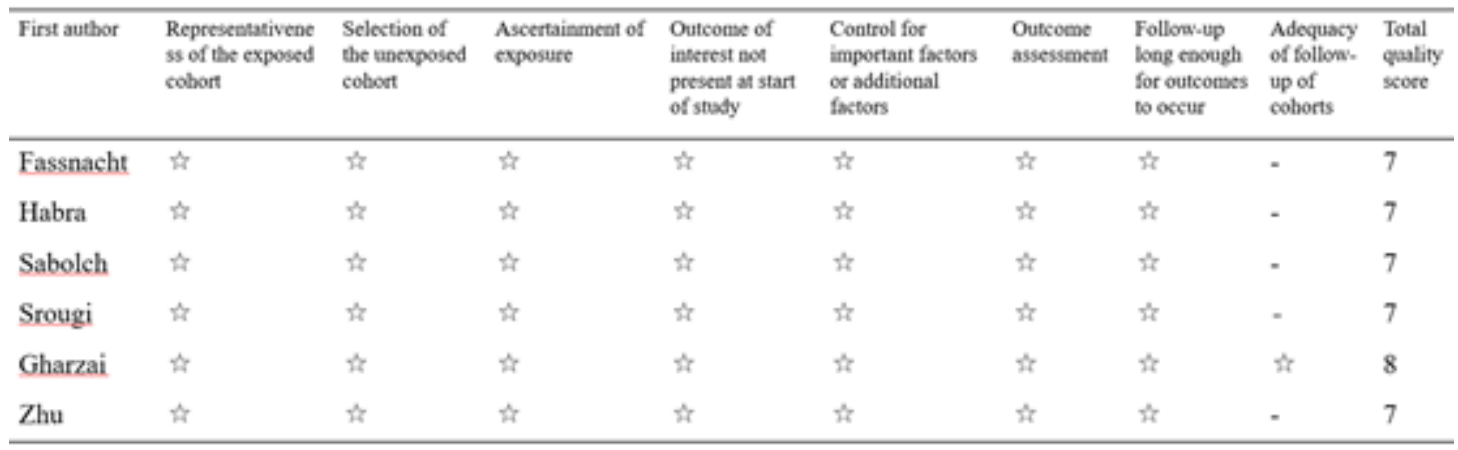

\section{Figures}
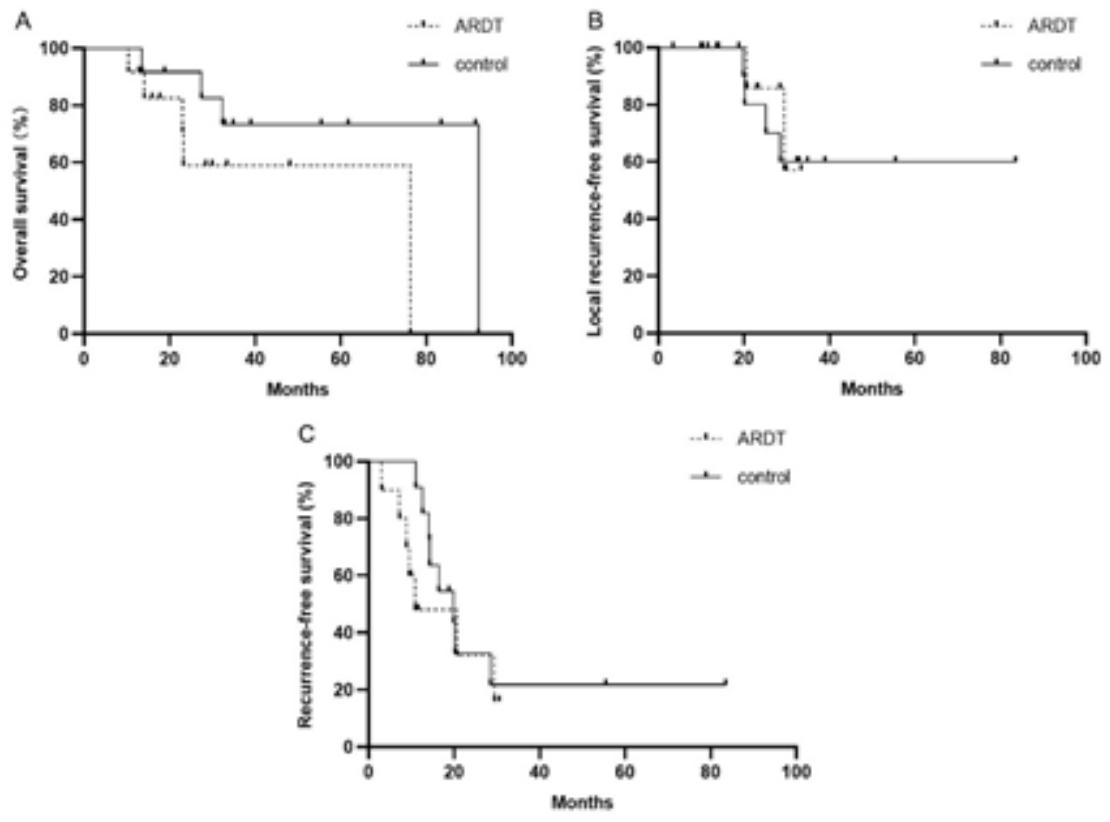

\section{Figure 1}

Kaplan-Meier analysis of overall survival (A), local recurrence-free survival (B), recurrence-free survival (C). 


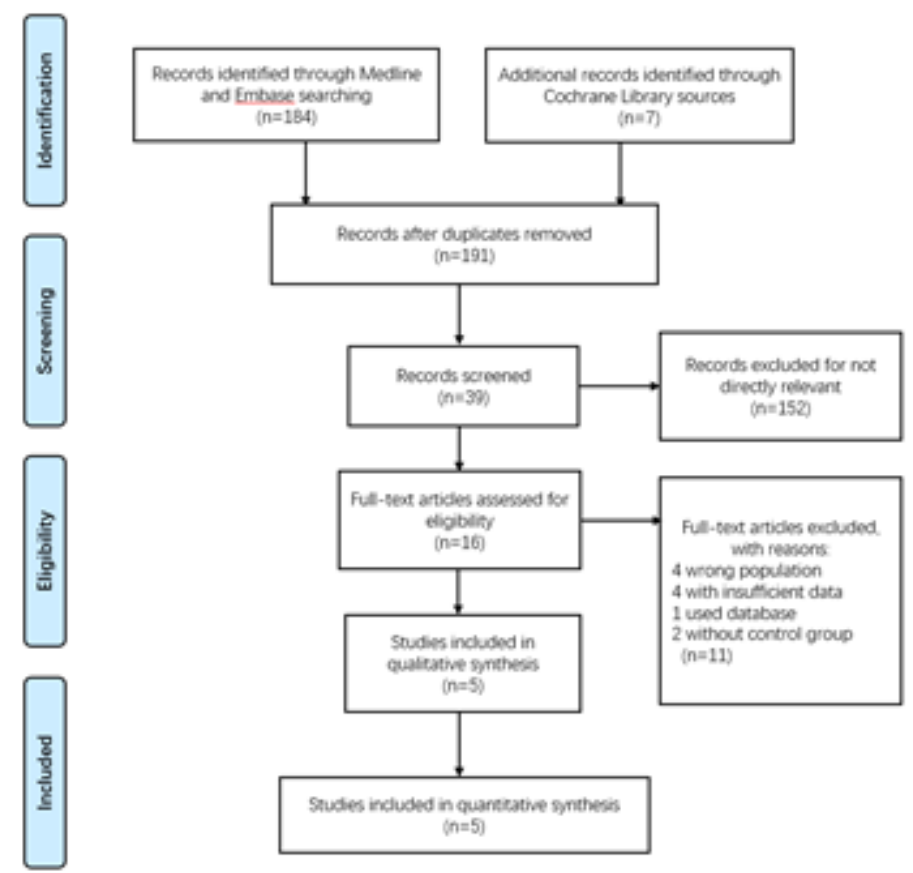

Figure 2

Study selection process of meta-analysis.

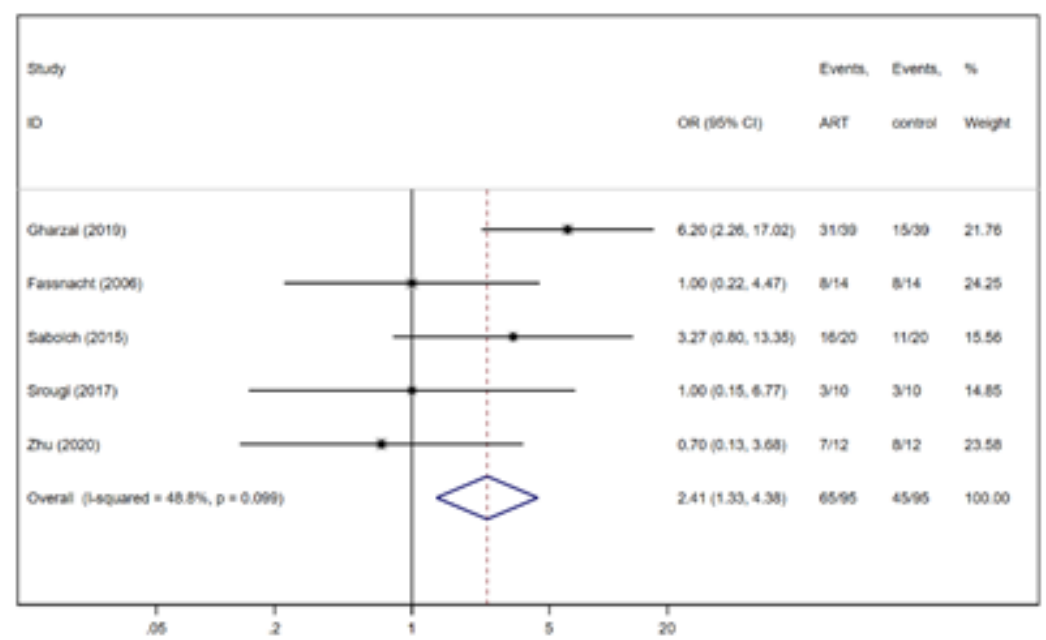

Figure 3

Forest plot of OS for ACC. 


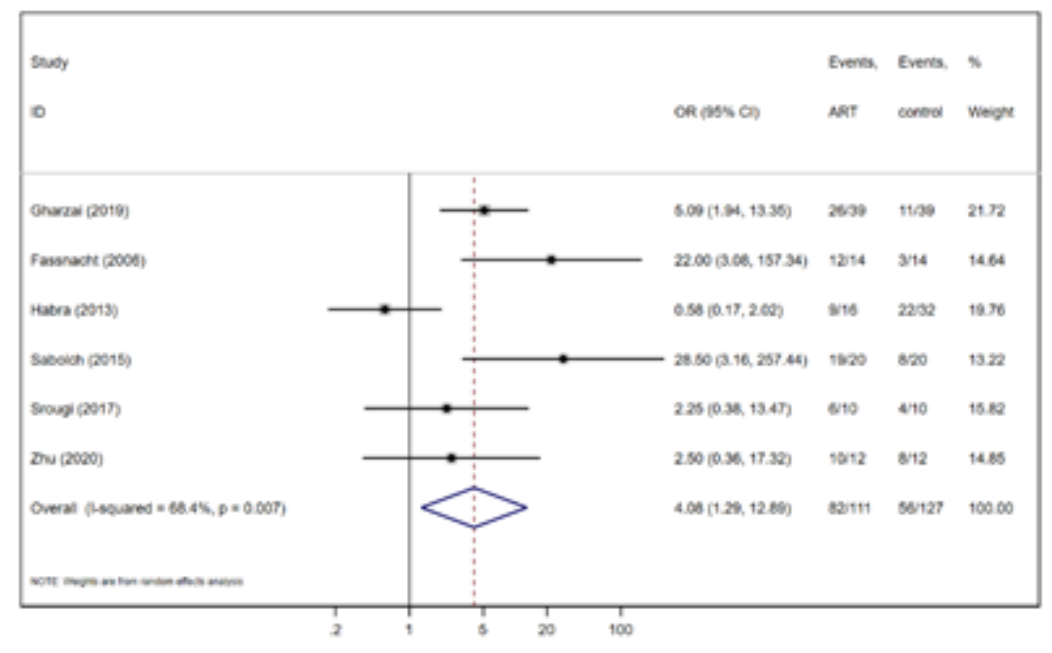

Figure 4

Forest plot of LRFS for ACC.

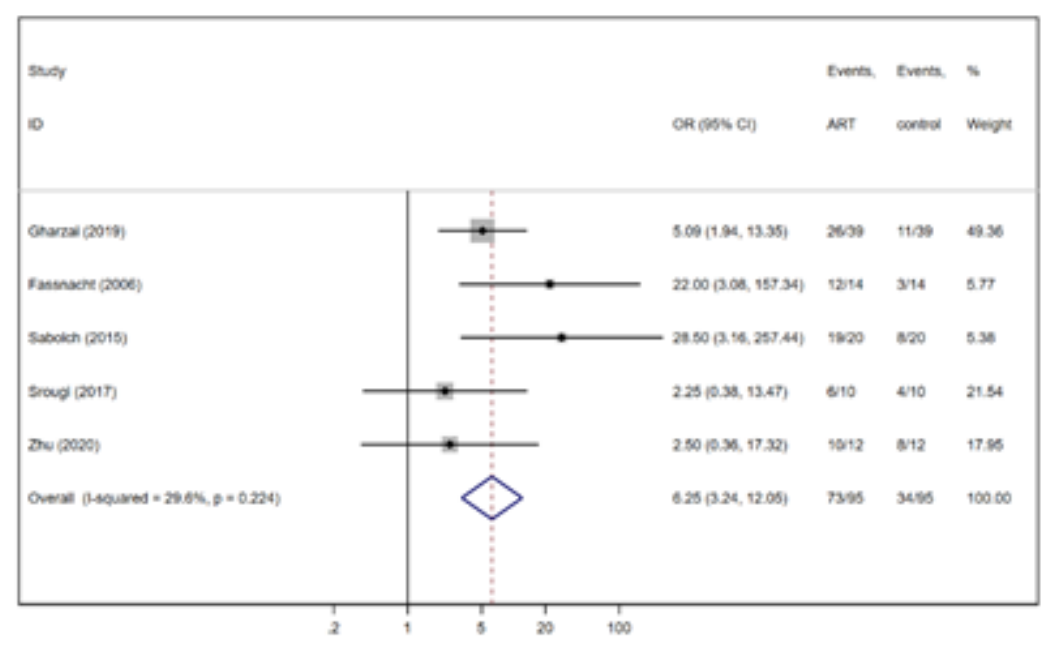

Figure 5

Forest plot of LRFS for ACC after sensitive analysis. 


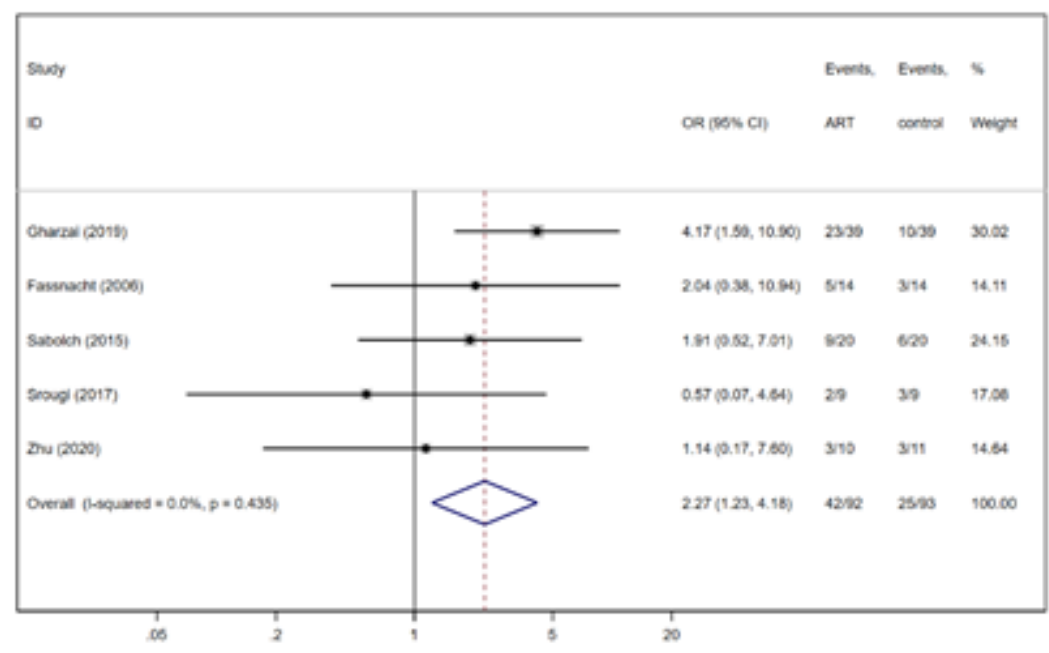

Figure 6

Forest plot of RFS for ACC.

\begin{tabular}{|c|c|c|}
\hline Author & & LRFS rate \\
\hline Zhu 2020 & $\longmapsto \quad 0-1$ & $83.3 \%(10 / 12)$ \\
\hline Srougi2017 & $\longmapsto$ & $60.0 \%(6 / 10)$ \\
\hline Sabolch 2017 & $\longmapsto$ & $95.0 \%(19 / 20)$ \\
\hline Habra 2012 & $\longmapsto$ & 56.3\% $(9 / 16)$ \\
\hline Fassnacht 2006 & $\longmapsto$ & $85.7 \%(12 / 14)$ \\
\hline Gharzai 2019 & $\longmapsto \bullet$ & $66.7 \%(26 / 39)$ \\
\hline 30 & 40 & 70 \\
\hline
\end{tabular}

Figure 7

Radiation dose and LRFS rate of included studies. 